\title{
Potential phytopharmaceutical constituents of Solanum trilobatum L. as significant inhibitors against COVID-19: Robust-binding mode of inhibition by molecular docking, PASS-aid bioactivity and ADMET investigations
}

Shanmugam Anandakumar ${ }^{a^{*}}$, Kannan Damodharan ${ }^{\mathrm{a}, \mathrm{b}}$, Eugene Wilson ${ }^{\mathrm{c}}$, Kasthuri Bai Narayanan $^{a}$, Ganesan Suresh ${ }^{\mathrm{a}}$, Kadarkarai Kanakavalli ${ }^{\mathrm{c}}$ and Manoharan Muthu Tamizh ${ }^{\mathrm{d}^{*}}$

${ }^{a}$ Yukai Care Solutions LLP, Chennai-600011, Tamil Nadu, India

${ }^{\mathrm{b}}$ Department of Organic and Bioorganic Chemistry, CSIR-Central Leather Research Institute, Chennai 600020, Tamil Nadu, India

${ }^{c}$ Central Council for Research in Siddha, Ministry of AYUSH, Chennai - 600106, Tamil Nadu, India

${ }^{\mathrm{d}}$ Department of Chemistry, Siddha Central Research Institute, Central Council for Research in Siddha, Chennai - 600106, Tamil Nadu, India

\section{ABSTRACT}

The novel coronavirus is better known as COVID-19 caused by Severe Acute Respiratory Syndrome Corona-Virus 2 (SARS-CoV-2) which initially outburst at Wuhan in China on December 2019 and spread very rapidly around the globe. Scientists from the global regions endeavours to still probe for detecting potential treatment and discover effective therapeutic drug candidates for this unabated pandemic. In our article, we reported the molecular docking, bioactivity score, ADME and toxicity prediction of the phytoconstituents of Solanum trilobatum Linn. such as Solanidine, Solasodine and $\alpha$ Solanine as potential inhibitors against the main protease $\left(\mathrm{M}^{\text {pro }}\right)$ of SARS-CoV-2 tropism. The molecular docking of Solanidine, Solasodine and $\alpha$-Solanine has revealed that it bounded deep into the active cavity site on the $\mathrm{M}^{\text {pro }}$. Further, the pharmacodynamics and bioactivity profile has confirmed that the molecules obeyed the Lipinski's rule and will be used as notably treasured lead drug candidates to pursue further biochemical and cell-based assays to explore its potential against COVID-19 pandemic. Thus, envisioning thoughtprovoking research certainly provide new leads for the global researchers.

KEYWORDS: Alkaloids; Molecular docking; Pharmacodynamics; Bioactivity profile; SARS-CoV-2

CONTACT ${ }^{a}$ Shanmugam Anandakumar, bioinfoanand@gmail.com, Yukai Care Solutions LLP, Chennai600011, Tamil Nadu, India; ${ }^{\mathrm{d}}$ Manoharan Muthu Tamizh, m.muthutamizh@gov.in, Siddha Central Research Institute, Central Council for Research in Siddha, Chennai - 600106, Tamil Nadu, India 


\section{Introduction}

The entire mankind across the globe is immensely suffering due to COVID-19 pandemic outbreak substantial morbidity and unprecedented mortality. Moreover, the death toll has reached more than six million and still its count increasing every day which indicates the ensuing perilous situation. Hence, there is an urgency to find potential and suitable drug candidates for this novel syndrome primarily affecting the respiratory system. Prior extensive studies shows that many targets are found for the treatment of COVID-19 disease, in which the glyco-protein resembling spike (S) glycoprotein plays a significant role for entering and spreading of highly pathogenic virus SARS-CoV-2 (Xia et al., 2020). Once this virus enters the human body, it strongly establishes and grows with an aid of another protein called Main protease $\left(\mathrm{M}^{\text {pro }}\right)$ enzyme (Hoffmann et al., 2020). Therefore, the prominent investigation is necessary towards the exploitation of this viral entry and growth in host cell through well-recognized lead potential bioactive antiviral agents is the present need for drug development. Gratifyingly, we have chosen such potential phytoconstituents of the Solanum trilobatum Linn. especially, the natural products including Solanidine, Solasodine, $\alpha-$ Solanine might be a resourceful inhibitor which was ascertained via in silico studies against COVID-19.

Solanum trilobatum Linn. a member of Solanaceae family, is a native plant of Asian countries specifically prevailing in India, Sri Lanka, Indonesia, Singapore, and Malaysia. Significantly, Solanum trilobatum Linn. is served in the form of soup to COVID-19 patients at Siddha COVID Care Centres (SCCC) and is part of the diet chart issued by the Ministry of AYUSH and Directorate of Indian Medicine and Homoeopathy, Government of Tamil Nadu (https://main.ayush.gov.in/event/guidelines-siddha-practitioners) (Ministry of AYUSH, Govt. of India, 2020). Prior studies indicate its vast therapeutic potential to serve as characteristic anticancer, antioxidant, anti-diabetic, anti-hepatocarcinogenesis, cytotoxicity and anti-inflammatory properties etc. (Mohan and Devi, 1996; Ganesan et al., 2017; Shilpha et al., 2015; Shahjahan et al., 2005; Jahan et a1., 2007; Ramar et al., 2015; Pandurangan et al., 2011). In Tamil vernacular language, it is popularly referred as Thoothuvelai and Singivalli. In Sanskrit, it is called as alarka. In the Ayurveda and Siddha system of medicine, their roots, fruits, and leaves are prescribed to heal various respiratory tract problems, including tuberculosis, asthma, sinusitis, tonsillitis, common cold, cough, pulmonary infections etc. (Govindan, 1999 and 2003; Mohanan et al., 1998). The leaf extract appears to 
increase male fertility and counteracts snake poison and cures lung sicknesses (Emmanuel et al., 2006).

The bioactivity of the herbs is based the following perspectives namely taste, potency, post digestive effect (PDE), individual action of the herbs and other characteristic properties (Uthamarayan, 2006). In fact, the taste of Solanum trilobatum Linn. is pungent and less bitter with hot potency that results in producing pungent flavour during post digestive period. It is indicated to tackle Aiyam (Phlegm), Vali (Air) and Azhal (Heat) humours. Moreover, its individual action includes stimulant, expectorant and tonic action (Mudaliar, 2006). According to the primal literature (Anand Kumar, 1975), Solanum trilobatum Linn. is used as a rejuvenator and consumed as greens which are capable of alleviating phlegmatic diseases. The leaves are used to treat hypogeusia, flower is an aphrodisiac, fruit pacifies all the three humours. Of course, root and creeper relieve cough, wheezing and phlegmatic afflictions respectively. In addition, the specific action of leaves alleviates dullness of the ear and its related disorders especially due to phlegm, cough, pruritus, ascites, dysbiosis, vitiated humours in unison and pricking pain in the body and provides strength to the body (Mudaliar, 2006)

In addition, the dried fruit is beneficial for vitiated phlegm, air and heat associated disorders as well as loss of appetite and hernia, whereas the ripe fruit useful for soothening vitiated humours, loosen the increase of phlegm in the bronchial tubes and catarrh and also used in toxic conditions. Most importantly the leaves decoction is indicated for bronchial asthma. If Solanum trilobatum Linn. is consumed as rejuvenator in the form of green leaves, root, dried fruits and pickles with cow ghee, it alleviates vitiated heat disorders and especially cures eye related disorders (Thiyagarajan, 2003).

The phytochemical screening of the genus Solanum including Solanum trilobatum Linn. characterized by several secondary metabolites, including glycoalkaloids, steroidalglycosides and saponins wherein, the most useful glycoalkaloids are Solanidine, Solasodine and $\alpha$-Solanine. Since, the toxic level of glycoalkaloids in human diet is still not confirmed (Hasanain et al., 2015). Predominantly, the identified chemical ingredients have numerous pharmacological activities such as antiviral (Lee et al., 2007; Sinani and Eltayeb, 2017), anticancer (Delporte et al., 1988; Choi and Koo, 2005; Emmanuel et al., 2006) and anti-diabetic activity (Sato, 1967). 
An abundanance of healing properties are found in the active components of Solanidine, Solasodine and $\alpha-$ Solanine from Solanum trilobatum Linn. This has been taken for investigation to study the interaction and binding affinity within the active site cavity of $\mathrm{M}^{\text {pro }}$ through molecular docking approach. Further the SwissADME were examined including the physiochemical properties, drug likeness and ADME of compounds. Subsequently, we have attempted to elicit their bioactivity profiles on the basis of PASS online property explorer and ultimately checked the toxicity of molecule using PreADMET server.

\section{Materials and Methods}

\subsection{Molecular Docking of S. trilobatum compounds against $M^{\text {pro }}$}

The molecular docking method was used to observe the interplay bonding pattern of compounds within the active site areas on the $\mathrm{M}^{\text {pro }}$ of SARS-CoV-2. The recently determined native structure of the main protease or $\mathrm{M}^{\text {pro }}$ (PDB: 6LU7) was taken from RCSB Protein Data Bank. The shape of the protein was refined and removed all non-standard residues including water by using the protein preparation protocol of Discovery Studio (DS) 2019.

Eventually, the binding sites were created on $\mathrm{M}^{\text {pro }}$ by means of selecting the compounds via DS to obtain the binding site dimensions of XYZ -5.03, 7.84, 78.25 for docking. Accordingly, the compounds were prepared and minimized. Further, conformations of compounds were generated with maximum 225 numbers of conformations and conformations of separate isomers had been also created inside the threshold of $20.0 \mathrm{kcal} / \mathrm{mol}$ relative energy. The CHARMm force field was applied to minimize the compounds with 1000 steps of steepest descent (SD) algorithm. The root mean square deviation (RMSD) $<1.0$ $\AA$ were taken into consideration as duplicates as the higher demanded RMSD value will reduce the number of ligands poses lower back and an RMS gradient 0.001 (Rao et al., 2007).

Diller and Merz have developed a high-throughput docking algorithm known as LibDock which includes the polar and apolar functions as "Hotspots". The molecules which bypass the implemented filters were docked with the crystal structure of $\mathrm{M}^{\text {pro }}$ (PDB: 6LU7). The chosen compounds were docked to explore and compare interacting amino acids. The docking parameters were kept default and CHARMm force field was employed to predict the docking score of the protein-ligand complexes. Ligand-receptor minimization was performed during docking by in situ cross-docking approach that changed into accomplished on the complexes to remove any ligand van der Waals clashes prior scoring and calculating 
binding energy (Diller and Merz Jr, 2001). The 5000 steps of SD with unfastened movement of atoms in the binding site sphere have been used at the time of minimization.

The binding strength of protein-ligand complexes had been calculated from the unfastened energies of the complex and free energies of protein and the ligand using CHARMmforce field and implicit salvation technique (Tirado-Rives and Jorgensen, 2006). Compounds were prepared for use of docking and other programs by way of the usage of DS, mainly for those which require a 3-dimensional (3D) coordinates and biological ionization and tautomerization states. While analyzing receptor-ligand interactions and other regions which miles essential to correctly prepare the ligands. The special protonation states, isomers and tautomers usually have specific 3D geometries and binding characteristics.

\subsection{ADMET prediction}

The physicochemical properties, lipophilicity, water solubility, pharmacokinetics of molecules were analysed through the SwissADME sever (Daina et al., 2017). The Absorption Distribution Metabolism and Elimination (ADME) properties for the represented molecules was used to calculate and assess the drug ability to filter the ligand molecules at an early stage of identifying the reason for its activity

\subsection{Biological activity profile}

The PASS online (http://www.pharmaexpert.ru/passonline/) was used to predict the biological activity including pharmacological effects, mechanism of action, toxic and adverse effects, interaction with metabolic enzymes, transporters and influence on gene expression, etc (Lagunin et al., 2000).

\subsection{Toxicity prediction}

The prior enters the small molecule into the human and animal models confirming the amount of their tolerability. Toxicity prediction of molecules were done using Pre-ADMET (https://preadmet.bmdrc.kr/) which is online based tool for predicting ADME data and building drug-like library using in silico method (Lee, 2003).

\section{Results}

\subsection{Docking Studies}


The identified compounds of Solanidine, Solasodine and $\alpha$-Solanine were docked with the $\mathrm{M}^{\text {pro }}$ (PDB ID: 6LU7) using LibDock module of Discover studio 2019 and hundreds of pose conformations were generated for each compound within the binding pocket. The best pose was screened for further analysis based on their LibDock score. The docking summary of compounds with target proteins of 6LU7 is depicted in Table 1. 
Table 1. Docking simulation summary of phytoconstituents of Solanum trilobatum Linn. with Mpro (PDB ID :6LU7)

\begin{tabular}{|c|c|c|c|c|c|}
\hline S. No & Compounds & Structure & $\begin{array}{l}\text { LibDock } \\
\text { Score }\end{array}$ & $\begin{array}{l}\text { Binding Energy } \\
(\mathrm{Kcal} / \mathrm{mol})\end{array}$ & Interacting Residues \\
\hline 1. & Solanidine & & 114.81 & -69.01 & $\begin{array}{l}\text { HIS 41, CYS 44, MET 49, PRO 52, TYR 54, } \\
\text { PHE 140, LEU 141, ASN 142, GLY 143, SER } \\
\text { 144, CYS 145, HIS 163, HIS 164, MET 165, } \\
\text { GLU 166, HIS 172, ASP187, ARG } 188 \text { and GLN } \\
189\end{array}$ \\
\hline 3. & $\alpha$-Solanine & & 172.63 & -203.00 & $\begin{array}{l}\text { HIS 41, MET 49, LEU 50, LEU 141, ASN142, } \\
\text { GLY 143, SER 144, CYS 145, HIS 163, HIS 164, } \\
\text { MET 165, GLU 166, LEU 167, PRO 168, HIS } \\
\text { 172, ARG 188, GLN 189, THR 190, ALA } 191 \\
\text { and GLN } 192\end{array}$ \\
\hline
\end{tabular}




\subsection{Binding mode of $S$. trilobatum compounds with $M^{\text {pro }}(P D B: 6 L U 7)$}

The binding affinity of Solanidine towards the active site of $\mathrm{M}^{\text {pro }}$ (PDB: 6LU7) was predicted by performing molecular docking analysis (Figure 1a and 1b). The Solanidine $\mathrm{M}^{\text {pro }}$ complex was established by one hydrogen bond and one carbon hydrogen bond. The hydrogen bond formed between H30 atom on hydroxyl group of Solanidine with the oxygen atom on carbonyl group of ASP 187 (2.0 $)$ and the carbon - hydrogen bond formed between methyl hydrogen of ARG 188 (2.2 $\AA$ ) and O1 atom on hydroxyl group of Solanidine (Figure 1c and 1d) respectively. Further the seven hydrophobic interactions (Alkyl \& $\pi$-Alkyl) were placed in residues of HIS 41, MET 49, CYS 145, HIS 163 and MET 165 for improving the physicochemical properties of Solanidine. Moreover, CYS 44, PRO 52, GLN 189, GLU 166, LEU 141, PHE 140, HIS 172, ASN 142, SER 144, GLY 143, HIS 164 and TYR 54 of active

site residues were involved in the van der Waals interaction in Solanidine $-\mathbf{M}^{\text {pro }}$ complex (Figure 1d) 


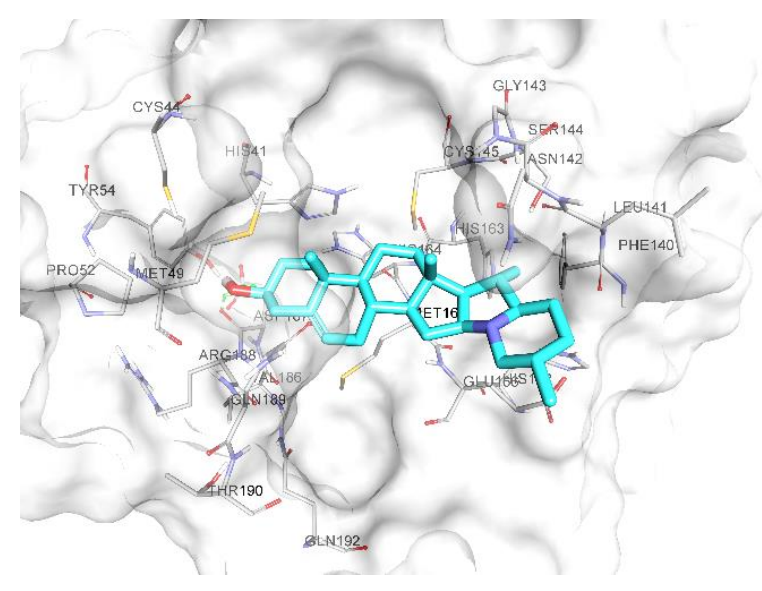

(a)

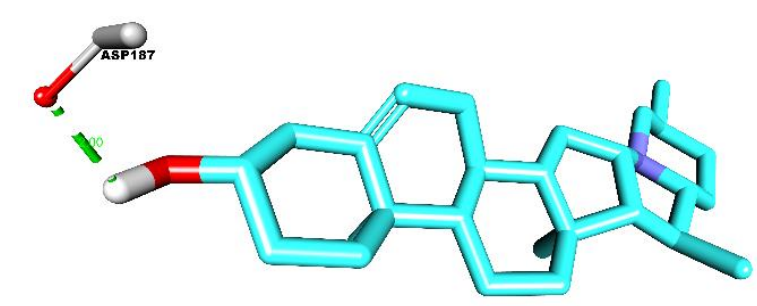

(c)

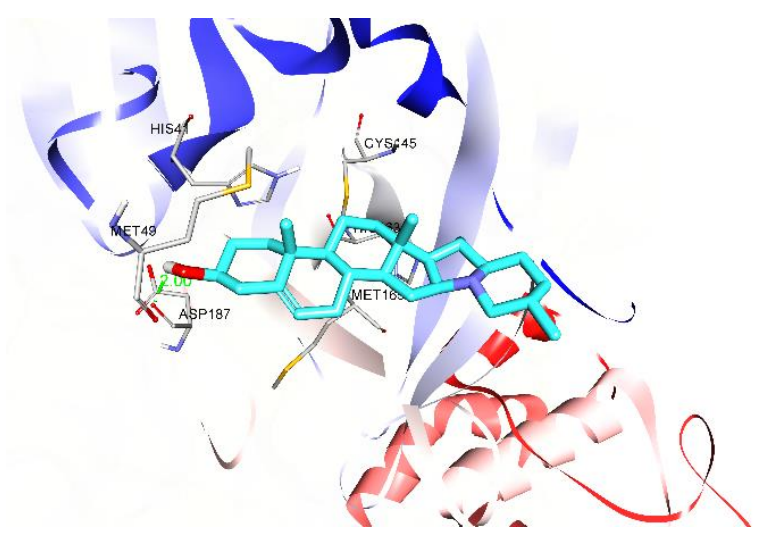

(b)

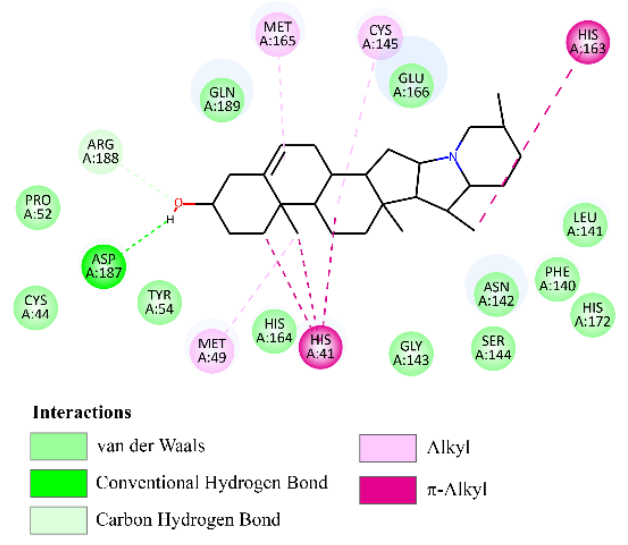

(d)

Figure 1 (a) Clear view of Solanidine and the substrate-binding pocket shown as surface illustration. The Solanidine is shown as cyan sticks and the crucial residues of $\mathbf{M}^{\text {pro }}$ are shown as atom colour sticks. (b) The secondary structure view of $\mathrm{M}^{\text {pro }}$ - Solanidine complex (Blue ribbon indicates the $N$-terminal and red ribbon indicates the $C$-terminal). (c) Stereo illustration of the interaction between Solanidine and the $\mathrm{M}^{\text {pro }}$. Residues and the Solanidine are shown as sticks, and the hydrogen bond between Solanidine and $\mathrm{M}^{\text {pro }}$ is shown by the green dashed line. (d) The $2 \mathrm{D}$ view of Solanidine-M $\mathrm{M}^{\text {pro. }}$. It obviously demonstrates conventional hydrogen bond, carbon - hydrogen bond, hydrophobic and van der Waals interactions present in receptor-ligand complex. 
The molecular docking of Solasodine at the active site of $\mathrm{M}^{\mathrm{pro}}$ (PDB:6LU7) revealed that it fits into the active site cavity perfectly (Figure $\mathbf{2 a}$ and $\mathbf{2 b}$ ). In this Solasodine-- $\mathbf{M}^{\text {pro }}$ complex, two hydrogen bonds were found. The nitrogen atom of Solasodine is protonated during the docking simulation and resulted in the formation of hydrogen bond interaction between H31 atom attached with nitrogen of Solanidine and carbonyl oxygen (O) of ASP 187 $(2.0 \AA)$ residue. Interestingly, the electrostatic attraction with bond distance $(2.8 \AA)$ was also observed between nitrogen on solasodine and carboxylic group of GLU 166 to maintain stability of $\mathrm{M}^{\text {pro }}$ - Solasodine complex. Another hydrogen bond formed between hydroxyl hydrogen atom (H32) of Solasodine and carbonyl group of PHE 140 (2.8 $\AA$ ) residue (Figure 2c and 2d). The three carbon - hydrogen bonds were found to support to the $\mathrm{M}^{\text {pro }}$ Solanidine complex. The methylene hydrogen $\left(\mathrm{CH}_{2}\right)$ present in the piperidinyl unit, adjacent to nitrogen atom of Solasodine was involved in the formation of carbon - hydrogen interaction with carbonyl O atom of PHE 140 (2.5 $\AA$ ) and carboxylic group of GLU 166 residue $(2.5 \AA)$ respectively. The methine proton of ARG 188 interacted with hydroxyl oxygen $(\mathrm{O} 2)$ atom of Solanidine through carbon - hydrogen interaction with bond distance of 2.5 A. Moreover, six hydrophobic interactions (Alkyl, $\pi$-Alkyl) were formed in the residue of HIS 41, MET 49, CYS 145 and MET 165 which improved the lipophilicity of Solasodine. Furthermore, the active site residues of the CYS 44, TYR 54, PRO 52, GLN 189, HIS 164, HIS 163, HIS 172, LEU 141, ASN 142, SER 144 and GLY 143 were participated in van der Waals interactions (Figure 2d). 


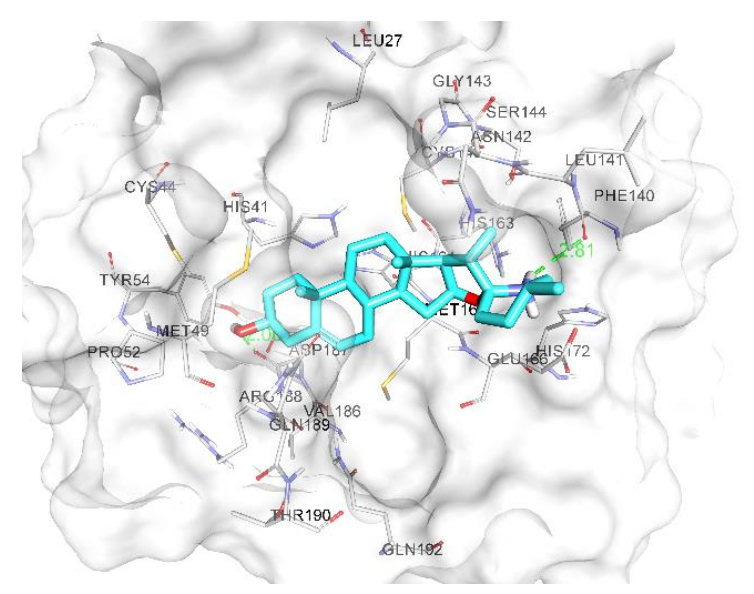

(a)

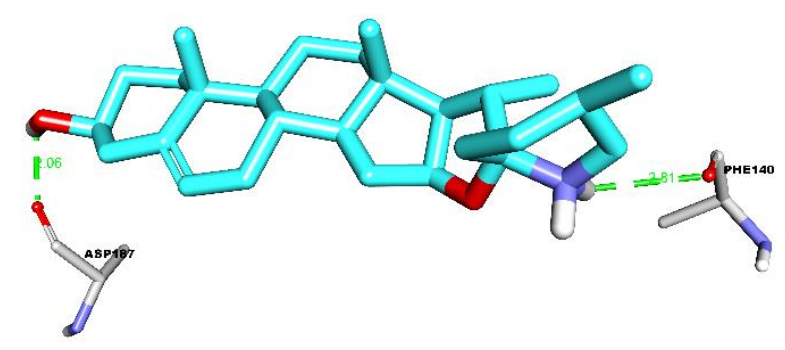

(c)

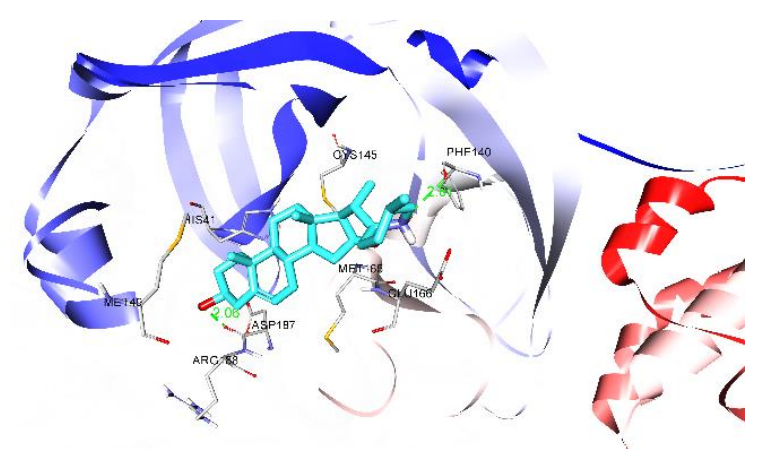

(b)

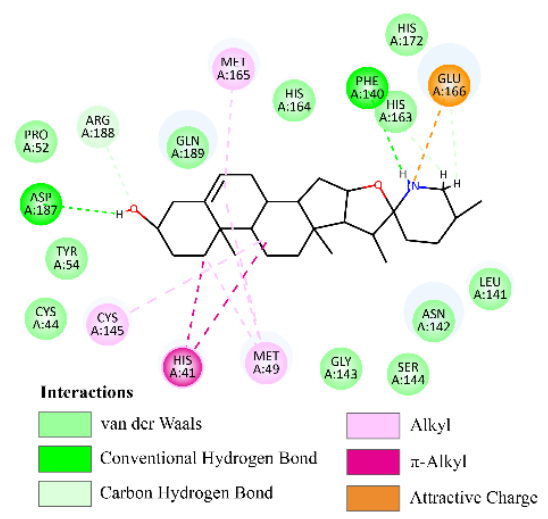

(d)

Figure 2(a) Detailed view of Solasodine and the substrate-binding pocket, shown in surface representation. The Solasodine is shown as cyan sticks, and the crucial residues of $\mathbf{M}^{\text {pro }}$ are shown as atom colour sticks (Blue ribbon indicates the $N$-terminal and red ribbon indicates the $C$-terminal). (b) The secondary structure view of $\mathrm{M}^{\text {pro }}$ - Solasodine complex (Blue ribbon indicates the $N$-terminal and red ribbon indicates the $C$-terminal). (c) Stereo view of the interaction between Solasodine and the $\mathrm{M}^{\text {pro }}$. Residues and the Solasodine are shown as sticks, and the hydrogen bond between Solasodine and $\mathrm{M}^{\text {pro }}$ is shown by the green dashed line. (d) The 2D view of Solasodine-M $\mathrm{M}^{\text {pro }}$. It clearly shows hydrogen bonds, carbon hydrogen bonds, attractive charge, hydrophobic and van der Waals interactions.

The molecular docking of $\alpha-$ Solanine with $\mathrm{M}^{\text {pro }}$ (PDB: 6LU7) revealed that the glycoside moiety of $\alpha$-Solanine was bound deep into the substrate-binding pocket of the receptor. Figure 3a and $\mathbf{3 b}$ clearly shows that $\alpha-$ Solanine has established with eight hydrogen bonds (Figure 3c and 3d). Amongst, the $\alpha$-Solanine has donated five hydrogen bonds to the $\mathrm{M}^{\mathrm{pro}}$ whilst, accepted three hydrogen bonds from the receptor. The tertiary nitrogen of $\alpha-$ 
Solanine have protonated to quaternary nitrogen during the docking simulation and resulted in the formation of hydrogen bond between $\mathrm{H} 78$ atom on tertiary $\mathrm{N}$ atom and $\mathrm{O}$ atom of carbonyl group of GLN 189 (1.9Å) residue. Further, the hydrogen atoms (H123, H125 and H127) on $\beta-D-$ glucopyranoside ring of $\alpha$-Solanine displayed hydrogen bonds between $S$ atom of CYS $145(2.7 \AA)$, O atom of carbonyl group of GLN 189 (2.7 $\AA$ ) and HIS 164 (1.7 $\AA$ ) respectively. Also, the H113 atom on ethanolic group of $\beta-D$-galactopyranoside ring formed hydrogen bond with carboxylic group of GLU 166 residue (1.9 $\AA$ ). The $\mathrm{H}$-acceptor chemistry of ligand from protein, $\alpha$-Solanine have accepted the three hydrogen bonds from $\mathrm{M}^{\text {pro }}$ to reinforce the protein - ligand complex viz (i) amine hydrogen of ASN 142 interacted with 059 atom of $\beta-D$-galactopyranoside ring $(2.5 \AA)$, (ii) amide hydrogen of GLU 166 interacted with ether link $\mathrm{O} 4$ atom which bridges $\alpha-\mathrm{L}-$ rhamnopyranosyl ring and $\beta-\mathrm{D}-$ galactopyranoside ring with the distance of $2.1 \AA$ and (iii) amide hydrogen of THR 190 interacted with hydroxyl group (O59 atom) of $\alpha$-L-rhamnopyranosyl ring (2.5 $⿱$ A). Also, six carbon - hydrogen bonds were formed between this ligand and protein complex. In addition, the following residues such as LEU 50, 165 and LEU 167 were gained substantial contribution with five hydrophobic interactions (Alkyl) for maintaining the physiochemical properties of $\alpha$-Solanine (Figure 3d). Further, the van der Waals interactions occurred in the respective residues as HIS 41, MET 49, LEU 141, GLY 143, SER 144, HIS 163, HIS 172, ALA 191 and GLN 192 (Figure 3d). 


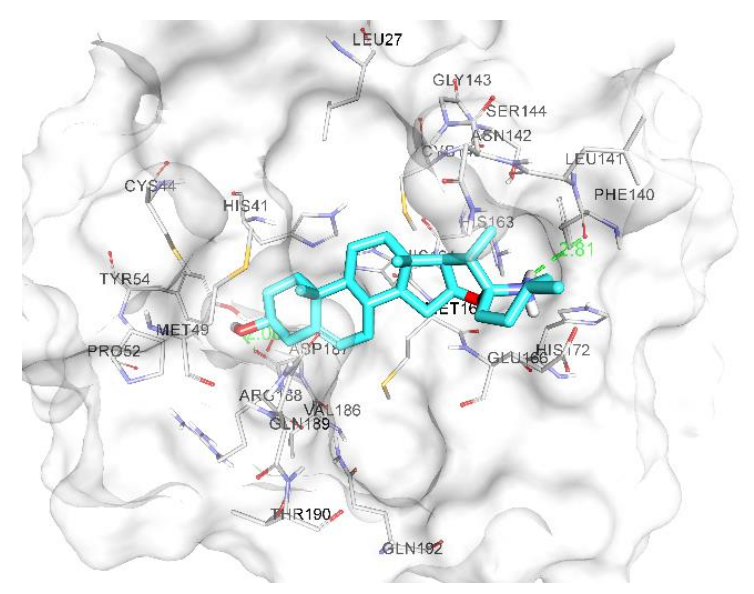

(a)

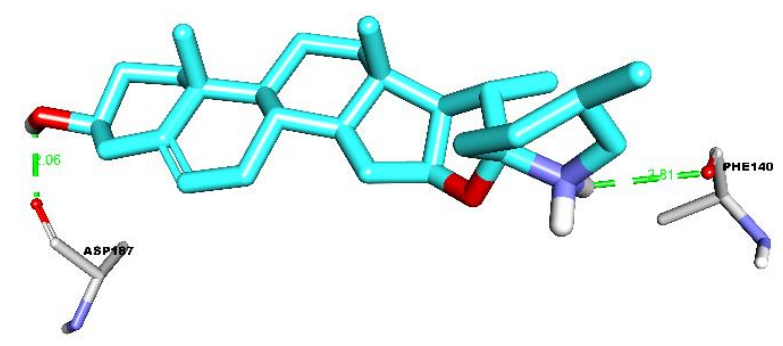

(c)

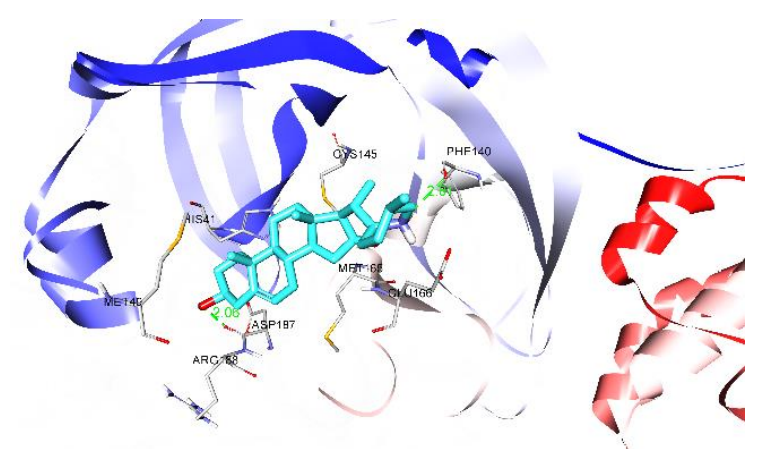

(b)

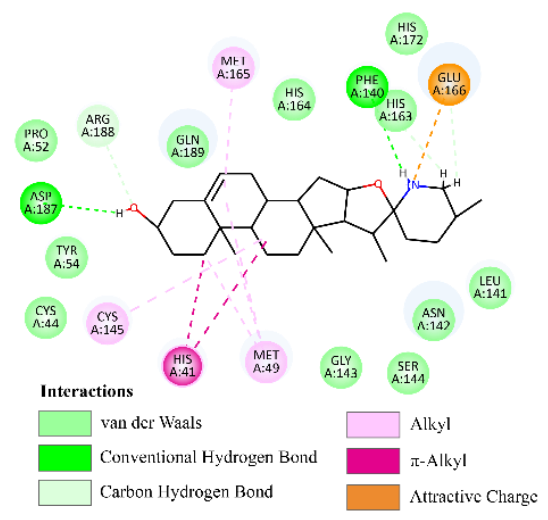

(d)

Figure 2(a) Detailed view of Solasodine and the substrate-binding pocket, shown in surface representation. The Solasodine is shown as cyan sticks, and the crucial residues of $\mathrm{M}^{\text {pro }}$ are shown as atom colour sticks (Blue ribbon indicates the $N$-terminal and red ribbon indicates the $C$-terminal). (b) The secondary structure view of $\mathrm{M}^{\text {pro }}$ - Solasodine complex (Blue ribbon indicates the $N$-terminal and red ribbon indicates the $C$-terminal). (c) Stereo view of the interaction between Solasodine and the $\mathrm{M}^{\text {pro }}$. Residues and the Solasodine are shown as sticks, and the hydrogen bond between Solasodine and $\mathrm{M}^{\text {pro }}$ is shown by the green dashed line. (d) The $2 \mathrm{D}$ view of Solasodine-Mpro. It clearly shows hydrogen bonds, carbon hydrogen bonds, attractive charge, hydrophobic and van der Waals interactions.

\subsection{ADME prediction}

Physiochemical and drug likeliness properties of compounds were analyzed by using Swiss-ADME portal. Lipinski's rule of 5 is generally utilized by pharmaceutical chemists in drug design and development to expect oral bioavailability of capability lead or drug molecules. The ADME predictions which include physicochemical properties, lipophilicity, water solubility and pharmacokinetics were obtained from Swiss-ADME respectively. 


\subsubsection{Physiochemical properties}

The physiochemical properties and drug likeliness of compounds were obtained from SwissADME server. Solanidine have showed 29 number of heavy atoms, 2 hydrogen bond acceptors and 1 hydrogen bond donors, while the molar refractivity is 126.20 and topological polar surface area (TPSA) of the molecule is found to be $23.47 \AA^{2}$. The physiochemical properties of the Solasodine associated with 30 heavy atoms, 3 hydrogen bond acceptors, 2 hydrogen bond donors, molar refractivity is 127.23 and topological polar surface area (TPSA) of the molecule is found to be $41.49 \AA^{2}$. The 30 number of heavy atoms, 16 hydrogen bond acceptors and 9 hydrogen bond donors were found in the $\alpha$-Solanine. The molar refractivity is 222.19 and topological polar surface area (TPSA) of the molecule is found to be $240.69 \AA^{2}$. The compounds were showed drug likeliness according to the Lipinski's Rules. Medicinal chemistry parameters have proved to be of no PAINS alert and violate Brenk's laws with few alerts of being an isolated alkene with molecular weight of greater than 350 . The synthetic accessibility of rate was noted 5.59 for Solanidine, 6.56 for Solasodine and 9.47 for $\alpha-$ Solanine.

\subsubsection{Lipophilicity}

The lipophilicity of the Solanidine has showed such as iLOGP is 4.50, XLOGP3 is 6.09, WLOGP is 5.27, MLOGP is 5.41, SILICOS-IT is 3.83 and Consensus P0/W is 5.02. Whereas in Solasodine, iLOGP is 4.32, XLOGP3 is 5.39, WLOGP is 4.91, MLOGP is 4.94, SILICOS-IT is 3.97 and Consensus P0/W is 4.71. In the $\alpha$-Solanine lipophilicity were noted, iLOGP is 4.71 , XLOGP3 is 1.81 , WLOGP is -0.23 , MLOGP is -1.14 , SILICOS-IT is -1.93 and Consensus $\mathrm{P} 0 / \mathrm{W}$ is 0.65 .

\subsubsection{Water Solubility}

The water solubility properties were calculated for each compound by different solubility method. Solanidine ESOL $(\operatorname{LogS})$ is -6.14 , solubility $7.21 \times 10^{-7} \mathrm{~mol} / \mathrm{L}$ and poorly soluble; Ali (LogS) is -6.36 , solubility $4.33 \times 10^{-7} \mathrm{~mol} / \mathrm{L}$ and poorly soluble; SILICOS-IT $(\operatorname{LogS})$ is -4.14 , solubility $7.19 \times 10^{-5} \mathrm{~mol} / \mathrm{L}$ and moderately soluble. Solasodine ESOL $(\operatorname{LogS})$ is -5.80 , solubility $1.58 \times 10^{-6} \mathrm{~mol} / \mathrm{L}$ and moderately soluble; Ali $(\operatorname{LogS})$ is -6.02 , $9.65 \times 10^{-7} \mathrm{~mol} / \mathrm{L}$ and poorly soluble, SILICOS-IT $(\operatorname{LogS})$ is -4.80 , solubility of $1.57 \times 10^{-5}$ $\mathrm{mol} / \mathrm{L}$ and moderately soluble. $\alpha-$ Solanine ESOL $(\operatorname{LogS})$ is -5.83 , solubility $1.46 \times 10^{-6}$ 
$\mathrm{mol} / \mathrm{L}$ and moderately soluble; Ali $(\mathrm{LogS})$ is -6.48 , solubility $3.28 \times 10^{-7} \mathrm{~mol} / \mathrm{L}$ and poorly soluble; SILICOS-IT $(\operatorname{LogS})$ is 0.75 , solubility $5.57 \mathrm{~mol} / \mathrm{L}$ and soluble.

\subsubsection{Pharmacokinetics}

According to Christopher A. Lipinski (1997), the pharmacokinetics of molecular properties is essential for a drug's in the human body (ADME; Absorption, distribution, metabolism and excretion). Pharmacokinetic data predicted for Solanidine was found to have high gastrointestinal absorption (GI), does not inhibit CYP1A2, CYP2C19, CYP2C9, CYP2D6 and CYP3A4 cytochromes. Skin permeation kinetics $(\operatorname{LogKp})$ is $-4.40 \mathrm{~cm} / \mathrm{s}$. Solasodine was found with high gastrointestinal absorption (GI), does not inhibit CYP1A2, CYP2C19, CYP2C9, CYP2D6 and CYP3A4 cytochromes. Skin permeation kinetics (LogKp) is $-5.00 \mathrm{~cm} / \mathrm{s}$. Whereas, $\alpha-$ Solanine have low gastrointestinal absorption (GI), neglects blood brain barrier permeant (BBB), acts as a P-gp substrate, does not inhibit CYP1A2, CYP2C19, CYP2C9, CYP2D6 and CYP3A4 cytochromes. Skin permeation kinetics (LogKp) is -10.31 $\mathrm{cm} / \mathrm{s}$.

\subsection{Biological activity prediction}

The PASS online (Prediction of Activity Spectra for Substances) is web-based application for the prediction of the biological activity of compounds based on their structural formulation for more than 4000 types of biological activity with frequent accuracy rate is above $95 \%$. The biological activity profiles were obtained based on their structural formula of the compounds from the PASS web browser. The pharmacological consequences and mechanisms of movement was calculated by probability of a chemical compound to be active $(\mathrm{Pa})$ or inactive $(\mathrm{Pi})$ based on evidence for activity already exceeds threshold $(\mathrm{Pa}>P \mathrm{i}$, by means of default). The probability threshold $\mathrm{Pa}>0.7$ were taken for the given Solanum trilobatum Linn compounds. The Table 2 shows the combined results of predicted biological activity for Solanidine, Solasodine and $\alpha$-Solanine together. 
Table 2. Predicted and important biological activity of Solanidine, Solasodine and $\alpha$-Solanine in PASS online sever

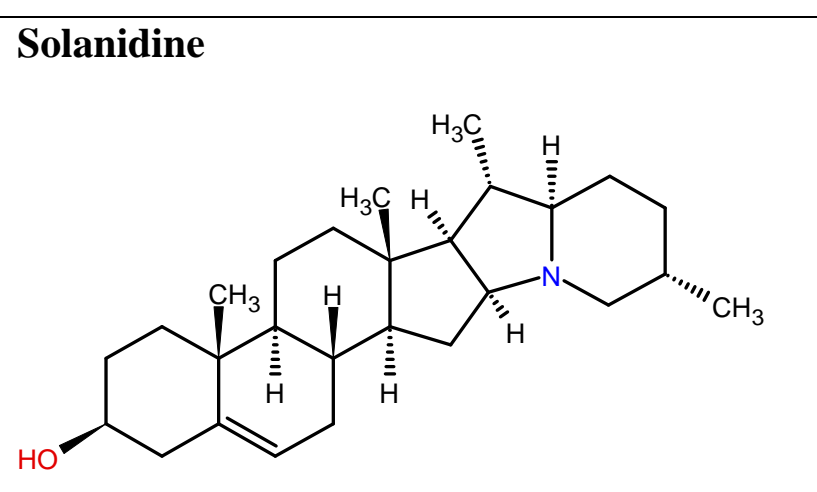

Solasodine

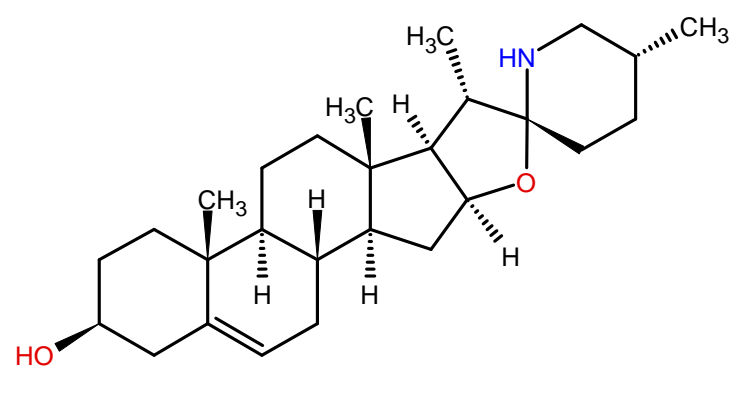

$\alpha$-Solanine

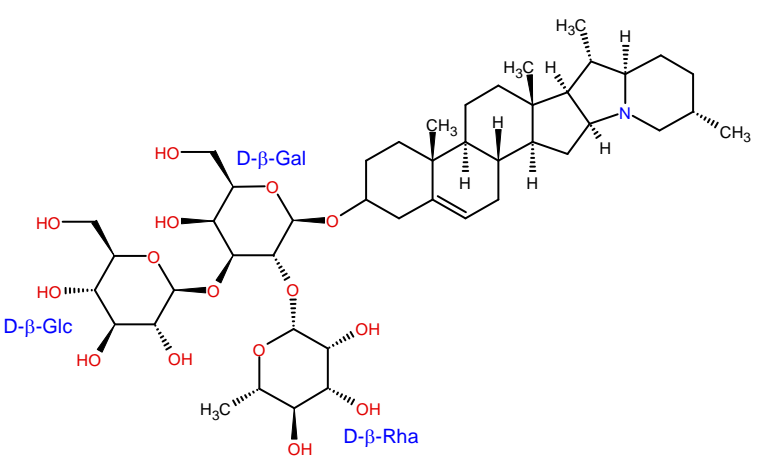

Pa $\quad P$ Activity

$0,929 \quad 0,002$ Cholesterol antagonist

$0,921 \quad 0,004 \quad$ Antiinflammatory

$0,896 \quad 0,003 \quad$ Antiprotozoal (Leishmania)

$0,914 \quad 0,005 \quad$ Antineoplastic

0,869 0,016 CDP-glycerol

glycerophosphotransferase inhibitor dehydrogenase (NADP+) inhibitor

0,790 0,005 Cholesterol antagonist

0,851 0,005 CYP3A4 inducer dehydrogenase (NADP+) inhibitor

$\begin{array}{lllllllll}\mathbf{0 , 7 9 9} & 0,002 & \text { CYP17 inhibitor } & 0,785 & 0,003 & \text { UGT1A4 substrate } & 0,832 & 0,005 & \text { CYP3A inducer } \\ \mathbf{0 , 7 9 3} & 0,003 & \begin{array}{l}\text { Acetylcholine neuromuscular } \\ \text { blocking agent }\end{array} & 0,776 & 0,003 & \text { CYP17 inhibitor } & 0,838 & 0,012 & \text { CYP2H substrate }\end{array}$




\begin{tabular}{|c|c|c|c|c|c|c|c|c|}
\hline 0,782 & 0,011 & $\begin{array}{l}\text { Alkylacetylglycerophosphatase } \\
\text { inhibitor }\end{array}$ & 0,749 & 0,008 & $\begin{array}{l}\text { Glyceryl-ether } \\
\text { monooxygenase inhibitor }\end{array}$ & 0,831 & 0,010 & $\begin{array}{l}\text { G-protein-coupled receptor } \\
\text { kinase inhibitor }\end{array}$ \\
\hline 0,796 & 0,037 & CYP2C12 substrate & 0,729 & 0,005 & $\begin{array}{l}\text { Antineoplastic (lung } \\
\text { cancer) }\end{array}$ & 0,831 & 0,010 & $\begin{array}{l}\text { Beta-adrenergic receptor kinase } \\
\text { inhibitor }\end{array}$ \\
\hline 0,738 & 0,010 & CYP3A inducer & 0,733 & 0,012 & Oxidoreductase inhibitor & 0,820 & 0,005 & $\begin{array}{l}\text { Glyceryl-ether monooxygenase } \\
\text { inhibitor }\end{array}$ \\
\hline 0,731 & 0,003 & $\begin{array}{l}\text { DELTA14-sterol reductase } \\
\text { inhibitor }\end{array}$ & 0,718 & 0,003 & Diuretic inhibitor & 0,813 & 0,002 & $\begin{array}{l}\text { Dolichyl- } \\
\text { diphosphooligosaccharide- } \\
\text { protein glycotransferase } \\
\text { inhibitor }\end{array}$ \\
\hline 0,738 & 0,010 & CYP3A4 inducer & 0,723 & 0,008 & UGT1A substrate & 0,822 & 0,015 & $\begin{array}{l}\text { Alkenylglycerophosphocholine } \\
\text { hydrolase inhibitor }\end{array}$ \\
\hline $\mathbf{0 , 7 3 7}$ & 0,011 & CYP3A5 substrate & 0,721 & 0,011 & CYP3A inducer & 0,796 & 0,004 & Hepatoprotectant \\
\hline 0,729 & 0,004 & UGT1A4 substrate & 0,716 & 0,005 & $\begin{array}{l}\text { Antineoplastic (breast } \\
\text { cancer) }\end{array}$ & 0,767 & 0,009 & Membrane integrity antagonist \\
\hline 0,734 & 0,012 & Oxidoreductase inhibitor & 0,709 & 0,014 & CYP3A5 substrate & 0,737 & 0,011 & CYP3A5 substrate \\
\hline
\end{tabular}

$P \mathrm{a}=$ Predicted activity; $P \mathrm{i}=$ Predicted inactivity 


\subsection{Toxicity prediction}

In-silico toxicity of compounds were checked with the help of PreADMET tool. AMES toxicity test was used to identify that a compound is mutagenic or not, the Solanidine showed as mutgen whereas the non-mutagen found in Solasodine and $\alpha$-Solanine. Carcinogenic profile also exposed that all compounds were non-carcinogenic in Mouse whereas carcinogenic in Rat except $\alpha$-Solanine. The hERG inhibition showed low risk in Solanidine and Solasodine whilst ambiguous in the $\alpha$-Solanine. The toxicity predictions indicated that the compound as anticipated to be secure (Table 3).

Table 3 PreADMET Toxicity prediction of Solanum trilobatum Linn phytoconstituents

\begin{tabular}{llllll}
\hline S. No. & Compounds & Ames Test & Carcinogenicity & hERGinhibition \\
\cline { 3 - 4 } & & & & \\
& & & & \\
\hline $\mathbf{1}$ & Solanidine & mutagen & negative & positive & low risk \\
$\mathbf{2}$ & Solasodine & non-mutagen & negative & positive & low risk \\
$\mathbf{3}$ & a-Solanine & non-mutagen & negative & negative & ambiguous \\
\hline
\end{tabular}

\section{Discussion}

Currently, the drug discovery for SARS-CoV-2 pandemic is looked upon anxiously in global perspective, owing to this unprecedented outburst and unattainable drug or other resources to prevent this disease. However, some of the repurposed synthetic drugs includes lopinavir, ritonavir, chloroquine and hydroxychloroquine derivatives which are used towards emergency; despite the fact that they may be irrelevant for patients with comorbidities like diabetes, hypertension and cardiac diseases (Aanouz et al., 2020; Rajib et al., 2020; Chorin et al., 2020). Therefore, the current need is to provide favourable alterative drug aspirants from therapeutically harmless phyto pharmaceuticals to facilitate the development of protective medicines to overcome the above lacunae. We envisage utilizing the phytopharmaceutical constituents from Solanum trilobatum Linn. owing to its valuable natural antiviral activity.

Hence, we analyzed such active ligands as antagonist against main protease enzyme $\left(\mathrm{M}^{\text {pro }}\right)$ by docking calculations which has provided the outstanding in silico estimations of the binding mode and the binding affinity of putative ligand molecules $-\mathrm{M}^{\text {pro }}$ complexes based on LibDock score along with least binding energy values. The best LibDock score of 114.81, 
113.969 and 172.63 were noted for the Solanidine, Solasodine and $\alpha$-Solanine complexes respectively. Correspondingly, the least binding energy of $-69.01 \mathrm{kcal} / \mathrm{mol}, 101.63 \mathrm{kcal} / \mathrm{mol}$ and $-203.00 \mathrm{kcal} / \mathrm{mol}$ calculated for each complex. In the binding mode, the putative ligands interacted with the strong hydrogen bonds interactions within the active site regions of the protein. Totally, the 21 hydrogen bonds (conventional and non-conventional) were identified in the Ligand $-\mathrm{M}^{\text {pro }}$ complexes. Amongst, the 2 hydrogen bonds found in Solanidine $-\mathrm{M}^{\text {pro }}$ complex, 5 in Solasodine $-\mathrm{M}^{\text {pro }}$ complex and 14 in $\alpha-$ Solanine $-\mathrm{M}^{\text {pro }}$ complex. Also, the attractive charge (electrostatic bond) was identified in the Solasodine $-\mathrm{M}^{\text {pro }}$ complex which gives additional strength to maintain the stability of protein - ligand complex. Moreover, the increasing hydrophobic interactions is a prime consideration for lead optimization, as this often calls for improving ligand molecular weight, rotatable bonds, and lipophilicity, all affecting the ADMET properties of ligands (Mullard, 2014). The significant numbers of hydrophobic interactions were identified in Solanidine, Solasodine and $\alpha$-Solanine which sufficiently improves the ADMET properties of the ligands. The active site residues like HIS 41, CYS 44, MET 49, PRO 52, TYR 54, TYR 54, PHE 140, LEU 141, HIS 163, HIS 164, GLU 166, HIS 172, ARG 188, GLN 189, ALA 191 and GLN 192 have commonly involved in the van dar Waals interaction which enlighten the molecules that are highly complementary.

The molecular descriptors of molecules had been examined concomitantly followed the Lipinski's rule of 5. The phyto compounds viz Solanidine and Solasodine fascinatingly abides by the characteristic property of molecular weight (MW) as within $500 \mathrm{Da}$. As per the rule, the lower molecular weight containing drug candidates $(<500 \mathrm{Da})$ is able to transport easily in drug movement, whilst, the $\alpha$-Solanine showed molecular mass is 868 Da. Obviously, some of the controlled drug release devices are available for a higher molecular weighted drug samples. Obviously, some of the natural products such as everolimus ${ }^{\mathrm{III}}(\mathrm{MW}=$ $958 \mathrm{Da})$, sirolimus ${ }^{\mathrm{III}}(\mathrm{MW}=914 \mathrm{Da})$, ivermectin $(\mathrm{MW}=875 \mathrm{Da})$ and paclitaxel $(\mathrm{MW}=854$ Da) are used as orally administered drugs with higher molecular weight (Tyagi et al., 2020). Moreover, nearly 500 drugs and clinical candidates have reported with MW ranging from 500 to $2000 \mathrm{Da}$ (Doak et al., 2014). From this observation, we can identify that our efficient drug component of $\alpha$-Solanine ( $\mathrm{MW}=854 \mathrm{Da}$ ) is negligible when compared to afore stated natural products and it can be conveniently utilized for the drug delivery system efficiently associated with classical approaches. 
Distinctly, the $\alpha$-Solanine have hydrophilic characteristics in nature, this is owed to the presence of three sugar units (pyranose) with nine hydroxyl groups may exhibit excellent antioxidant properties, and moreover the steroidal unit exist in $\alpha$-Solanine own hydrophobicity, in particular, the indolizidine unit also exhibiting numerous pharmacological activities such as anticancer, anti-TB, antioxidant, antimicrobial and anti-inflammatory etc. (Gómez et al., 2012). Therefore, all these representative valuable characteristics in this $\alpha-$ Solanine (gluco-steroidal framework) molecule apparently offered a major breakthrough which came into limelight. Altogether, during metabolism this existing privileged molecule may be act as promising pro-drug with amphipathic characteristics, it is worth to mention here that the existence of both hydrophilic and hydrophobic quality in a drug skeleton may used to facilitate the nature of various bio/pharmacological activities.

Apart from this, the number of hydrogen bond acceptors ( $\mathrm{O}$ and $\mathrm{N}$ atoms) and donors $(\mathrm{NH}$ and $\mathrm{OH})$ inside the selected phytoconstituents were revealed within Lipinski limits. Lipophilicity $(\log P)$ and the topological polar surface place (TPSA) values are important for the prediction of oral bioavailability for molecules to become a drug. The identified compounds have showed the LogP and TPSA values within the range, whereas it has proven as an excellent descriptor and characterizing drug absorption as represented in terms of an intestinal absorption, bioavailability and Blood-Brain-Barrier (BBB) penetration. Lipophilicity $(\log S)$ is at once related to the water solubility of a drug and it is described as a common solubility. The different values of lipophilicity $(\operatorname{LogS})$ were obtained fromthe ESOL (Delaney, 2004), Ali (Ali et al., 2012) and SILICOS-IT methods (http://silicos-it.be.s3website-eu-west-1.amazonaws.com/software/filter-it/1.0.2/filter-it.htm) which is specified as miscellaneous water solubility like soluble, moderate and poor witness in the compounds. Based on this facts, the phytopharmaceutical drugs of Solanum trilobatum Linn. are possibly being orally active as they obeyed Lipinski's rule of 5. The bioactivity rating of compounds is suggestive of slight interplay with all drug goals. The most promising compounds as in step with its high range of bioactivity scores have acknowledged to the compounds the way through PASS-aid bioactivity predictions, including Solanidine, Solasodine and $\alpha$-Solanine, which obviously exhibit promising biological properties like anticancer, anti-urease activity, antineoplastic, trypanocidal activity, antibacterial activity, immunomodulatory, antipyretic activities and also various effects on the central nervous system etc. (Cham et al., 1990; 
Nakamura et al., 1996; Quan et al., 2002; Lee et al., 2004; Chataing et al., 1998; Divyagnaneswari et al., 2008; Chauhan et al., 2011)

The pharmacokinetic activities together with absorption, distribution, metabolism, excretion indicated mainly in $\alpha$-Solanine which isn't always BBB permeate and it could acts as a P-gp substrate and also which does not inhibit CYP1A2, CYP2C19, CYP2C9, CYP2D6 and CYP3A4 cytochromes. In terms of the toxicity character of phytopharmaceutical compounds especially the $\alpha$-Solanine has confirmed that don't have the mutagenic and carcinogenic effects in the mouse and rat. Thereby, the phytopharmaceutical drugs of the Solanum trilobatum Linn typically to embark upon the current pandemic COVID-19.

Eventually, the obtained results suggests that these phytochemical constituents of Solanum trilobatum Linn functioning as potential protease inhibitors for SARS-CoV-2 owed to the presence of its valuable antiviral behaviour, which actively contributes for preventing the maturation of the $\mathrm{M}^{\text {pro }}$ and multiplication of virus within the cells. Presence of the aforesaid alkaloids were reconfirmed from the leaf extract of Solanum trilobatum Linn by HPTLC and other associated characterizations in our previous unpublished work. Obviously, this strategical process opens up the new avenues to promote these phytopharmaceutical sources will be a lead potential drug candidate for not only inhibit, but completely evacuate this SARS-CoV-2 tropism in upcoming generation.

\section{Acknowledgment}

The authors wish to thank the Yukai Care Solutions LLP for computer infrastructure facility. The author Kannan Damodharan thank CSIR-Central Leather Research Institute and Science and Engineering Research Board (SERB) for his (Project No.PDF/2017/001743) National Post Doctoral Fellowship (NPDF) award. The authors express the timely help provided by Dr. S. Vinayak, Research Associate (Siddha), CCRS, Chennai.

\section{Disclosure statement}

No potential conflict of interest was reported by the author(s). 


\section{Reference}

Aanouz, I., Belhassan, A., Khatabi, K. E. I., Lakhlifi, T., Idrissi, M. E. I., \& Bouachrine, M. (2020). Moroccan Medicinal plants as inhibitors of: Computational investigations. Journal of Biomolecular Structure \& Dynamics, 1-9. https://doi.org/10.1080/07391102.2020.1758790.

Al Sinani, S.S.S., Eltayeb, E.A. (2017) The steroidal glycoalkaloids solamargine and solasonine in Solanum plants. South African Journal of Botany, 112, 253-269. https://doi.org/10.1016/j.sajb.2017.06.002.

Ali, J., Camilleri, P., Brown, M. B., Hutt, A. J., Kirton, S. B. (2012) Revisiting the general solubility equation: in silico prediction of aqueous solubility incorporating the effect of topographical polar surface area. Journal of Chemical Information and Modeling, 52, 420428. https://doi.org/10.1021/ci200387c.

Anand Kumar, A. (1975) Theraiyar Kappiyam, First Edition, Pandit S. S. Anandam Research Institute of Siddha Medicine, Chennai - 600 017. pp 11.

Cham, B., Daunter, B. (1990) Solasodine glycosides. Selective cytotoxicity for cancer cells and inhibition of cytotoxicity by rhamnose in mice with sarcoma 180. Cancer Letters, 1990, 55, 209-220. https://doi.org/10.1016/0304-3835(90)90122-E.

Chataing, B., Concepcion, J., Lobaton, R., Usubillaga, (1998) A. Inhibition of Trypanosoma cruzi growth in vitro by Solanum alkaloids: a comparison with ketoconazole Planta Medica, 64, 31-36. https:// doi.org/10.1055/s-2006-957361

Chauhan, K., Sheth, N., Ranpariya, V. and Parmar, S. (2011) Anticonvulsant activity of solasodine isolated from Solanum sisymbriifolium fruits in rodents. Pharmaceutical Biology, 49(2): 194-199. https://doi.org/10.3109/13880209.2010.508499.

Choi, E., Koo, S. (2005) Anti-nociceptive and anti-inflammatory effects of the ethanolic extract of potato (Solanum tuberlosum). Food and Agricultural Immunology, 16, 29-39. https://doi.org/10.1080/09540100500064320.

Chorin, E., Dai, M., \& Shulman, E. (2020). The QT interval in patients with SARS-CoV-2 infection treated with hydroxychloroquine/azithromycin. MedRxiv, 55(3), 105923. https://doi.org/10.1101/2020.04.02.20047050v1. 
Daina, A., Michielin, O., \&Zoete, V. (2017). SwissADME: A free web tool to evaluate pharmacokinetics, drug-likeness and medicinal chemistry friendliness of small molecules. Scientific Reports, 7, 42717. https://doi.org/10.1038/srep42717.

Delaney, J. S. (2004) ESOL: Estimating Aqueous Solubility Directly from Molecular Structure. Journal of Chemical Information and Modeling, 44, 1000-1005. https://doi.org/10.1021/ci034243x.

Delporte, C., Backhouse, N., Negrete, R., Salinas, P., Rivas, R., Cassels, B. K., San Feliciano, A. (1998) Antipyretic, hypothermic and antiinflammatory activities and metabolites from Solanum ligustrinum Lood. Phytotherapy Research, 12, 118-122. https://doi.org/10.1002/(SICI)1099-1573(199803)12:2<118::AID-PTR207>3.0.CO;2-U.

Diller, D. J., Merz Jr, K. M. (2001) High throughput docking for library design and library prioritization. Proteins, 43, 113-124. https://10.1002/1097-0134(20010501)43:2<113::aidprot $1023>3.0 . \operatorname{co} ; 2-\mathrm{t}$.

Divyagnaneswari, M., Christybapita, D. and Dinakaran, R.M. 2008. Immunomodulatory activity of Solanum trilobatum leaf extracts in Oreochromis niloticus, pp. 221-234. In Bondad-Reantaso, M.G., Mohan, C.V., Crumlish, M. and Subasinghe, R.P. (eds.). Diseases in Asian Aquaculture VI. Fish Health Section, Asian Fisheries Society, Manila, Philippines.

Doak, B.C., Over, B., Giordanetto, F., Kihlberg, J. (2014) Oral druggable space beyond the rule of 5: Insights from drugs and clinical candidates. Chemistry \& Biology, 21, 1115-1142. https://doi.org/10.1016/j.chembiol.2014.08.013.

Emmanuel, S.; Ignacimuthu, S.; Perumalsamy, R.; Amalraj, T. (2006) Anti-inflammatory activity of Solanum trilobatum. Fitoterapia, 77, 611-612. https://doi.org/10.1016/j.fitote.2006.09.009.

Ganesan, K., Sukalingam. K., Xu, B. (2017) Solanum trilobatum L. Ameliorate Thioacetamide-Induced Oxidative Stress and Hepatic Damage in Albino Rats. Antioxidants, 6(3), 68. https://doi.org/10.3390/antiox6030068.

Gómez, L., Garrabou, X., Joglar, J., Bujons, J., Parella, T., Vilaplana, C., Clapés, P. (2012). Chemoenzymatic synthesis, structural study and biological activity of novel indolizidine and quinolizidine iminocyclitols. Organic \& Biomolecular Chemistry, 10(31), 6309-6321. https:// doi.org/10.1039/C2OB25943E. 
Govindan, S., Viswanathan, S., Vijayasekaran, V., Alagappan, R. (1999) A pilot study on the clinical efficacy of Solanum xanthocarpum and Solanum trilobatum in bronchial asthma. Journal of Ethnopharmacology, 66, 205-210. https://doi.org/10.1016/S03788741(98)00160-3.

Govindan, S., Viswanathan, S., Vijayasekaran, V., Alagappan, R. (2004) Further studies on the clinical efficacy of Solanum trilobatum in bronchial asthma. Phytotherapy Research, 18, 805-809. https://doi.org/10.1002/ptr.1555.

Hasanain, M., Bhattacharjee, A., Pandey, P., Ashraf, R., Singh, N., Sharma, S., Vishwakarma, A. L., Datta, D., Mitra, K., Sarkar, J. (2015) $\alpha$-Solanine induces ROSmediated autophagy through activation of endoplasmic reticulum stress and inhibition of Akt/mTOR pathway. Cell Death and Disease, 27, 6(8), e1860. https://doi.org/10.1038/cddis.2015.219.

Hoffmann, M., Kleine-Weber, H., Schroeder, S., Kruger, N., Herrler, T., Erichsen, S., Schiergens, T. S., Herrler, G., Nai-Huei, W., Nitsche, Andreas., Muller, M. A., Drosten, C., Pohlmann, S. (2020) SARS-CoV-2 Cell Entry Depends on ACE2 and TMPRSS2 and Is Blocked by a Clinically Proven Protease Inhibitor. Cell, 2, 271280.https://doi.org/10.1016/j.cell.2020.02.052.

Jahan, M.S., Vani, G., Shyamaladevi, C.S. (2007) Effect of Solanum trilobatum on hepatic drug metabolizing enzymes during diethylnitrosamine-induced hepatocarcinogenesis promoted by phenobarbital in rat. Hepatology Research, 37, 35-49. https://10.1111/j.1872034X.2007.00006.x.

Lagunin, A., Stepanchikova,A., Filimonov, D., Poroikov, V. (2000) PASS: prediction of activity spectra for biologically active substances. Bioinformatics, 8, 747-748. https://doi.org/10.1093/bioinformatics/16.8.747.

Lee, K. R., Kozukue, N., Han, J. S., Park, J. H., Chang, E. Y., Baek, E. J., Chang, J. S., Friedman, M. J. (2004) Friedman M. Glycoalkaloids and metabolites inhibit the growth of human colon (HT29) and liver (HepG2) cancer cells. Journal of Agricultural and Food Chemistry, 52, 2832-2839. https://doi.org/10.1021/jf030526d.

Lee, M. -H., Cheng, J. -J., Lin, C.-Y., Chen, Y.-J., Lu, M.-K. (2007) Precursor-feeding strategy for the production of solanine, solanidine and solasodine by a cell culture of 
Solanum lyratum. Process Biochemistry, 42, 899-903. https://doi.org/10.1016/j.procbio.2007.01.010.

Lee, S. K., Lee, I. H., Kim, H. J, Chang, G. S., Chung, J. E., No, K. T. (2003) The PreADME Approach: web-based program for rapid prediction of physicochemical, drug absorption and drug-like properties, Euro QSAR 2002 designing drugs and crop protectants: processes, problems and solutions, Blackwell Publishing, Massachusetts, USA, pp. 418-420.

Ministry of AYUSH, Govt of India (2020) Guidelines for Siddha Practitioners. https://main.ayush.gov.in/event/guidelines-siddha-practitioners.

Mohan, P. V., Devi, K. S., (1996) Cytotoxic potential of the preparations from Solanum trilobatum and the effect of Sobatum on tumor reduction in mice. Cancer Lett, 110, 71-76. https://10.1016/s0304-3835(96)04463-1.

Mohanan, P. V., Rao, J.M., Kutty, M. A. S., Devi, K.S. (1998) Cytotoxicity and anticarcinogenic activity of Sobatum. Biomedicine, 18, 106-111. https://doi.org/10.1016/S0304$\underline{3835(96) 04463-1 .}$

Mudaliar, K. S. M. (1988) Gunapadaam Porut Panbu Nool (Muthal Paagam), Second Edition, Directorate of Indian Medicine and Homoeopathy, Chennai - 600 106, pp. 534-537.

Mullard, A. (2014) New drugs cost US\$2.6 billion to develop. Nature Reviews Drug Discovery, 13, 877. https://doi.org/10.1038/NRD4507.

Nakamura, T., Komori, C., Lee, Y.-Y., Hashimoto, F., Yahara, S., Nohara, T., Ejima, A. (1996) Cytotoxic Activities of Solanum Steroidal Glycosides. Biological and Pharmaceutical Bulletin, 19, 564-566. https://doi.org/10.1248/bpb.19.564.

Pandurangan, A., Khosa, R.L., Hemalatha, S. (2011) Anti-inflammatory activity of an alkaloid from Solanum trilobatum on acute and chronic inflammation models. Natural Product Research, 25, 1132-1141. https://doi.org/10.1080/14786410903370783.

Quan, H.-J., Koyanagi, J., Ohmori, K., Uesato, S., Tsuchido, T., Saito, S. (2002) Preparations of heterospirostanols and their pharmacological activities. European Journal of Medicinal Chemistry, 37, 659-669. https:// doi.org/10.1016/s0223-5234(02)01386-7.

Rajib, I., Rimon, P., Archi, S., P., Nizam, U., Sajjadur, R., \& Abdulla, A., M. (2020). A molecular modeling approach to identify effective antiviral, of cyclin-cyclin-dependent 
kinase complex and blocks $\mathrm{S}$ phase progression in mammalian cells. The Journal of Biological Chemistry, 281(16), 10669-10681. https://doi.org/10.1074/jbc.M509233200.

Ramar, M., Manikandan, B., Marimuthu, P.N., Raman, T., Mahalingam, A., Subramanian, P., Karthick, S., Munusamy, A. (2015) Synthesis of silver nanoparticles using Solanum trilobatum fruits extract and its antibacterial, cytotoxic activity against human breast cancer cell line MCF 7. Spectrochimica Acta Part A, 140, 223-228. https://doi.org/10.1016/j.saa.2014.12.060.

Rao, S. N., Head, M. S., Kulkarni, A., LaLonde, J. M. (2007) Validation studies of the sitedirected docking program LibDock. Journal of Chemical Information and Modeling, 47, 2159-2171. https://doi.org/10.1021/ci6004299.

Sato. T., Glycemic effects of solanine in rats. (1967) The Japanese Journal of Pharmacology, 7, 652-658. https://doi.org/10.1254/jip.17.652.

Shahjahan, M., Vani, G., Shyamaladevi, C.S. (2005) Effect of Solanum trilobatum on the antioxidant status during diethyl nitrosamine-induced and phenobarbital promoted hepatocarcinogenesis in rat. Chemico-Biological Interactions, 156, 113-119. https://10.1016/j.cbi.2005.08.003.

Shilpha, L., Satish, L., Kavikkuil, M., Joe Virgin Largia, M., Ramesh, M. (2015) Methyl jasmonate elicits the solasodine production and anti-oxidantactivity in hairy root cultures of Solanum trilobatum L. Industrial Crops and Products, 71, 54-64. https://doi.org/10.1016/j.indcrop.2015.03.083.

Thiyagarajan, R., (2003) Theraiyar Yemaga Venba Part-I, Second Edition, Directorate of Indian Systems of Medicine \& Homoeopathy, Chennai - 600 106, pp. 37-38.

Tirado-Rives, J., Jorgensen, W. L. (2006) Contribution of conformer focusing to the uncertainty in predicting free energies for protein-ligand binding. Journal of Medicinal Chemistry, 49, 5880-5884. https://doi.org/10.1021/jm060763i.

Tyagi, M., Begnini, F., Poongavanam, V., Doak, B.C., Kihlberg, J (2020) Drug Syntheses Beyond the Rule of 5. Chemistry - A European Journal, 26(1), 49-88. https://doi.org/10.1002/chem.201902716. 
Uthamarayan, K. S. (2006) Siddhar Maruthuvanga Churukkam. Second Edition, Directorate of Indian systems of Medicine \& Homoeopathy, Chennai - 600 106, pp. 27-28.

Xia, S., Liu, M., Wang, C., Xu, W., Lan, Q., Feng, S., Qi, F., Bao, L., Du, L., Liu, S., Qin, C., Sun, F., Shi, Z., Zhu, Y., Jiang, S., Lu, L. (2020) Inhibition of SARS-CoV-2 (previously 2019-nCoV) infection by a highly potent pan-coronavirus fusion inhibitor targeting its spike protein that harbors a high capacity to mediate membrane fusion. Cell Research, 30, 343-355. https://doi.org/10.1038/s41422-020-0305-x. 